\title{
Performance of Electrical Power Network with Variable Load Simulation
}

\author{
Ahmed Al Ameri and Cristian Nichita \\ Groupe de Recherche en Electrotechnique et Automatique, GREAH Lab., University of Le Havre, France, \\ ahmed.al-ameri@etu.univ-lehavre.fr, cristian.nichita@univ-lehavre.fr
}

\begin{abstract}
Today's system operators face the big challenge of constructing simulation of systems that make efficient select of generation resources under variable load profiles. This paper describes IEEE five bus system modeling which simulated under Simulink. The real power load model designed to allow different load profile types (residential, commercial and industrial) connecting to load buses. The main purpose of this paper is to demonstrate the performance of power network based on load profile modeling as a means for enhance Distribution Network Operators (DNOs) decision in power systems. In this paper IEEE five bus system is used as a test bed. The results are shown with constant and variable load model. The results indicate the effectiveness of this flexible load profile model applied to the five bus system.
\end{abstract}

Keywords: power system analysis, load modelling, simulation, load profile, network modelling

\section{Introduction}

Deregulation of the electric energy systems and a progressive increase of the load play a key role in improving reliability and continuity of the electrical utility services they provide. Due to the complexity of load profile, many challenges facing electrical power system analysis, like, state estimation, load flow calculation, and network planning and operation. Distribution network operators (DNOs) report the performance of their network based on inadequate modelling approaches because large power systems are simply represented by a bulk load.

The hardest part for most DNOs is to determine the errors between estimated and actual load profile simulation. There is some of early-published state estimation studies describing the role of load profiling on the classical electrical power networks. Author in (Jardini et al., 2000), presents a statistic analysis of different load curves to obtain the representative curves of the most important customers. The measurements were performed for residential, commercial and industrial to determine customers' daily load profile. The fuzzy c-means (FCM) method is used for load profiling in (Chang et al., 2003). The load profile assignment performed by using customers' monthly energy usage data in two steps. After load profiling, the author used recognition technique to assign 500 customers. In (Kim et al., 2011), It was interesting to used load profile for energy diagnosis system. Different load profile data according to customer type (residential, commercial and industrial) obtained from metering devices. Consumption separation method was used to classify duration of load devices to a certain time interval (1 hour, 1 day, 1 week and 1 month).

On other hand, other authors modelling the optimal power flow control for dynamic grid where the load changes according to a profile. (Almeida et al., 2000) presents an algorithm to calculate a sequence of PrimalDual Interior Point optimization solutions under variable load conditions. This methodology to find optimal power flow based on two main steps: predictor, which estimate a new operating point for an increment in the load by linear approximation; and corrector step, which uses non-linear method to find the optimal solution to the new load level. A three load profile are used to find the optimal reactive power control. The daily load curves divided into several sequential levels to reduce operations of control devices switching. Voltage quality and power loss for different loads were consider as objective function for optimization techniques. Typical clustering method and heuristic iteration technique used the maximum load deviation (MLD) to decompose the load curve (Varga et al., 2015).

Other opportunities to improve planning and operation of modern network with/without distributed generation and storage under different load conditions are considered in a large number of articles recently (Hernando-Gil et al., 2016; Bazrafshan et al., 2017; Guggilam et al., 2016; Ma et al., 2016; Yi et al., 2016; Thrampoulidis et al., 2016; Geng et al., 2016, Li et al.,2016). This fact shows that this subject continues an interesting topic of research. In relation to modern network, a real-time classification and encoding of load profiles has been proposed in (Varga et al., 2015). The author presents software framework to manage the load profile at power system operation. The framework is based on artificial neural network as encoding engine and local hashing algorithm as classifier engine. A dynamic load profile was cluster and classify by multiresolution analysis (MRA) method (Bazrafshan et al., 2017). The ability of traditional methods in profiling load developed by MRA method for three key (large, 
volatility and uncertain smart metering data). A more flexible load profiling with less computation presented in this method by three main steps (decomposition, clustering and reconstruction).

Moreover, there has been an increasing amount of literature on the planning of modern power system's reliability. In active distribution systems, (Hernando-Gil et al., 2016) proposed methodology based on empirical load profile and time varying fault probabilities for reliability planning and risk estimation. The approach is developed to avoid the underestimation of network's performance at bulk supply points for more realistic estimation of customer interruptions.

Research has shown that installation of distributed generation and storage energy take more attention. Radial distribution networks with photovoltaic (PV) generation was tested to optimize the real power consumption of loads (Bazrafshan et al., 2017). The power management scheme developed to determine the optimal demand response schedule that accounts for variable real power injection by PV units. So that, the programmable loads provide opportunity to reduce the peak load in periods of inappropriate generation. In distribution feeders, load profile data and realistic photovoltaic (PV) generation are utilized to optimize its operation. The active and reactive power set points for $\mathrm{PV}$ was determine according to voltage regulation and a variety of objective functions (Guggilam et al., 2016). The proposed method leverage a linearized version to formulate a quadratic constrained quadratic program (QCQP) as direct applications to distribution networks with PV systems. The cost efficiency of the residential electricity consumption improved by load scheduling (Ma et al., 2016). The load scheduling framework based on fractional programing approach to develop a cost efficient for the demand side's day-ahead process and real-time pricing mechanism. The proposed algorithm considered the distributed energy resources and service free in their framework.

Finally, in attempt to improve the power system operation more effectively, energy storage systems installed (ESS) with/without distributed generation to the modern grid. Real load connecting to distribution networks has tested to schedule the ESS by Monte Carlo simulation (Yi et al., 2016). The optimization technique used for solving an ESS scheduling problem considering real load, variable wind energy sources and transmission line real time thermal rating (RTTR). The load shifting by optimize placement, sizing and control of energy storage system presented in (Thrampoulidis et al., 2016). The network topologies with regardless/regard of the load demand, generation capacities and line flow limitations effected the costs. A charging/discharging policy for the installed storage units formulated as slower time-scales.

However, investigating and modelling varying energy demands in various sectors (residential, commercial and industrial) will cause significant changes in planning, operation and control of power system. Most studies in load modelling and profiling into electrical power network have been carried out in a small number of area. This paper attempts to simulate the electrical network with different load profile, which can help DNOs to avert the underestimation of network's performance at bulk generation points for more actual estimation of customer interruptions. Load demand has been developed to determine the overall power flow and identify generation sources required to meet its increasing as well as exceeded rating elements in the network.

The rest of the paper is constructed as follows. Section 2 demonstrates the element of five node network modelling. Section 3 gives a brief overview of the load profile conception for different type of customers. Simulation is implemented and results are presented in Section 4. Conclusions are drawn in Section 5.

\section{Load Profile}

In power system, a load profile is a graph illustrating the variation in the demand/electrical load versus time. A load profile will vary according to temperature, holiday seasons and customer type (typical examples include residential, commercial and industrial),. DNOs use this information to plan how much electricity they will need to generate at any given period. These load curves are useful in the selection of generator units for providing electricity.

Direct metering devices such as smart grid meters, data logging sub-meters, utility meter load profilers and portable data loggers can determine load profiles. Real demand can be collected at strategic locations to analysis load performance, which is beneficial to both distribution and end-user customers looking for peak consumption (Geng et al., 2010). For most customers, based on meter reading schedules, consumption is measured on a monthly. Load profiles are used to convert the monthly consumption data into estimates of hourly or sub hourly consumption in order to determine the electrical utilities obligation. For each hour, these estimates are aggregated for all customers of an energy provider, and the aggregate amount is used as the total demand that must be covered by the utilities.

In this section, the detailed simulation of the load profile would be described. For brevity purpose, detailed simulation of the load profile for residential, commercial and industrial would be shown, covering the lower and higher side of the residential units. Simulation of three bus load are described concisely.

\subsection{Residential Consumers}

More than half of all electrical power is consumed by residences type, which vary in their daily activity patterns and the types of appliance they own. Load 
varies by time of day and year where its curve shape be function of consumer demand. Figure 1 illustrating how electrical demand characteristics varies over a day, or when house owners are using electrical power.

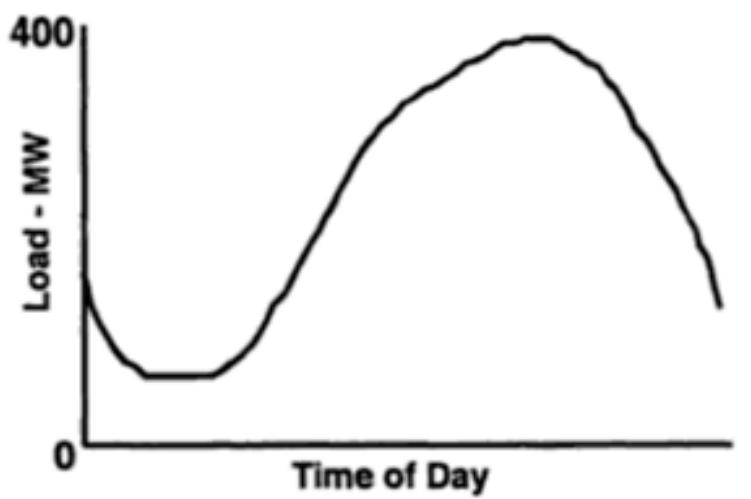

Figure 1. Example of residential consumption.

Due to the differences in electric appliances, the definition of the representative curves of a range is not an easy task to be done and the people habits rising to curves of shapes in the peak. In some countries, the residential energy consumption value is mainly due to refrigerator or freezer, whilst the water heating gives the curve peak where heater resistance take $8 \mathrm{~min}$ (Li et al., 2016). Therefore, it is very hard to characterize the peak demand because load pattern not fixed for all residential usage and depends on many factories such as weather, type of human work etc.

\subsection{Commercial and Industrial Consumers}

In commercial businesses load, small and large consumers having similar end uses to residential (cooling, heating etc) in addition to many need to commercial devices (office machinery, cash register, escalators etc). Figure 2 represents the electrical use of a commercial facility during 24 hours. The commercial load (office building, restaurants stores etc) shows a strong upward curve during summer (or winter) session because it depend heavily on cooling (or heating) systems (Lee, 2004).

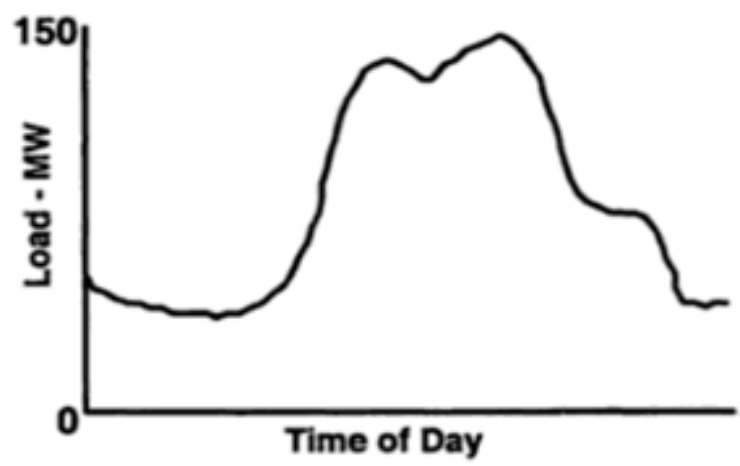

Figure 2. Example of commercial consumption.
Finally, industrial facilities and plants use electricity to variety of manufacturing applications such as compressor motors, heating systems etc. The industrial load profile does not vary as much through the day where it depend on work, weekends and break times. The peak demand of summer day for industrial consumption from utility system shown in Figure 3.

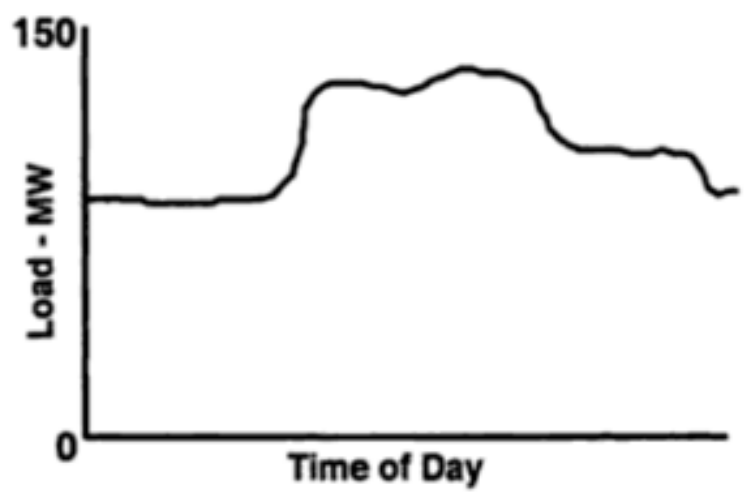

Figure 3. Example of industrial consumption.

\section{Node Network Modelling}

Throughout the electrical power network there are common buses that look like important branch points within the power grid. These buses operate at a defined voltage level and phase angle to forming the complex bus voltage. In general, three types of busses is consider in a power network, namely the slack bus, the generator bus and the load bus.

Figure 4 shows a single line diagram of a 5 bus system with two generating units, seven lines. Per unit system based on 100 MVA was considered for all parts of network. Four basic parts of the system are modelled: slack generator, PV control generator, transmission lines and load profiles. Figure 5 presents the simulation of the 5 bus network using Simulink.

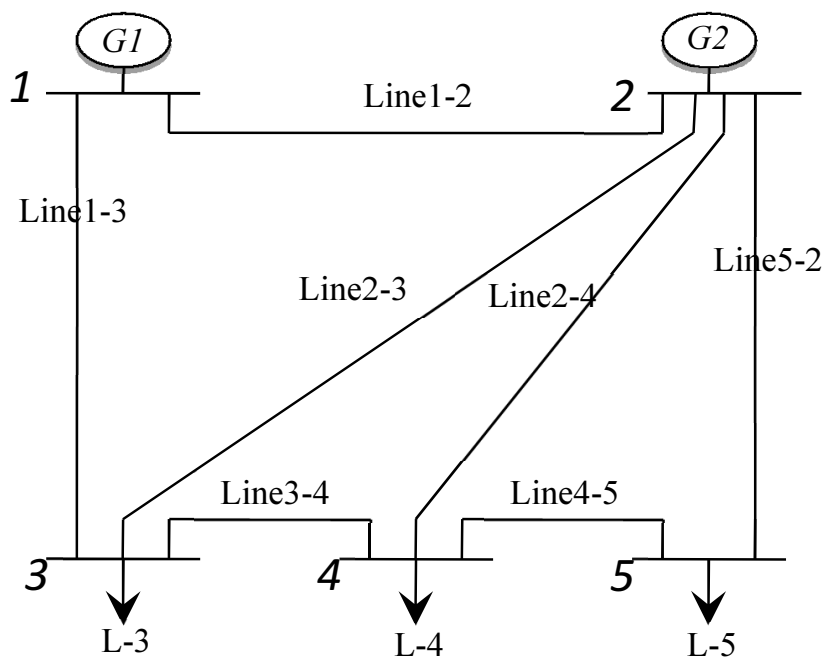

Figure 4. Single line diagram of 5 bus network. 


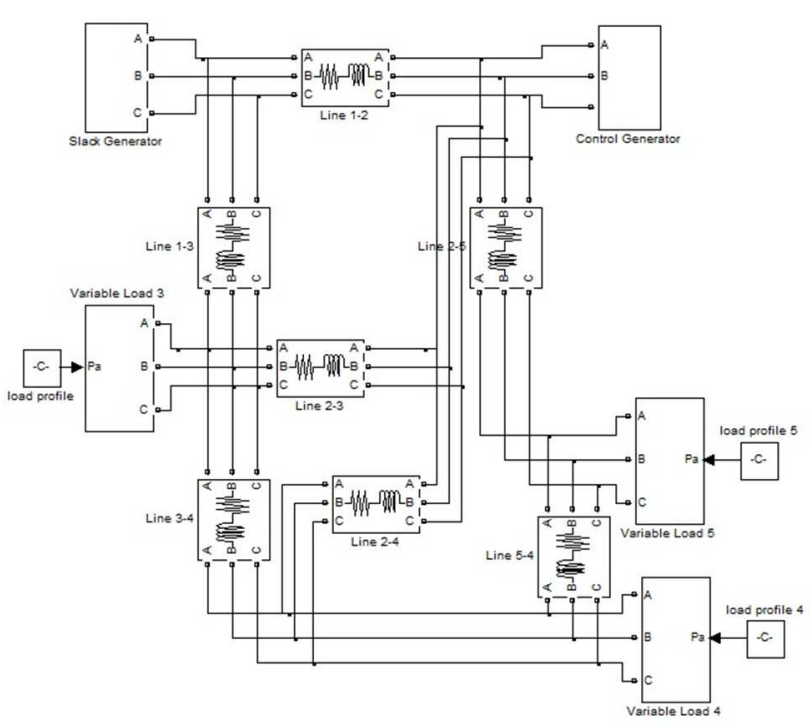

Figure 5. 5 bus network simulation.

All generation units, load demand and power loss calculated is shown in Table 1.

Table 1. Generation and load demand for IEEE 5 bus.

\begin{tabular}{|l|l|}
\hline Unit & Total (MW) \\
\hline Total Generation (PG2) & unlimited \\
\hline Total Generation (PG2) & 90 \\
\hline Max. Demand(PD3) residential & 45 \\
\hline Max. Demand(PD4) Commercial & 40 \\
\hline Max. Demand(PD5) Industrial & 60 \\
\hline
\end{tabular}

\subsection{Slack generator}

In a simulated power system, some quantities allowed to vary or swing to solve particular steady-state problem successfully. For that, there is only one slack generator has known voltage magnitude $|\mathrm{V}|$ and voltage phase (set to $1.0 \angle 0.0^{\circ}$ (per-unit)). In Figure 6, Psg and Qsg are the swing variables and obtained through the load flow solution as follows:

$$
S_{s g}=P_{s g}+j Q_{s g}
$$

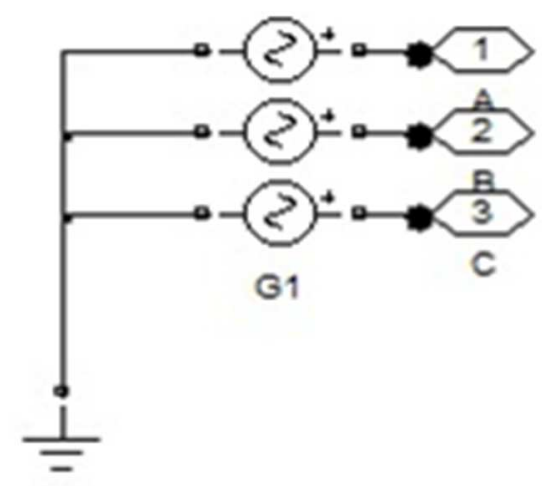

Figure 6. Slack generator simulator.

\subsection{Voltage Control Generator}

Generator buses or voltage controlled buses have inputs of the voltage magnitude corresponding to the generator voltage and real power $\mathrm{Pg}$ corresponds to its rating (Figure 7) . Generally, voltage controlled busses are connected to equipment used for voltage and VAR correction, such as static VAR correction systems, generators and shunt capacitors (Figure 8). It is calculated the reactive power generation Qg and phase angle of the bus voltage by load flow solution. In general, generator is modeled as a complex power injection at a specific bus (i) is

$$
s_{g}^{i}=p_{g}^{i}+j q_{g}^{i}
$$

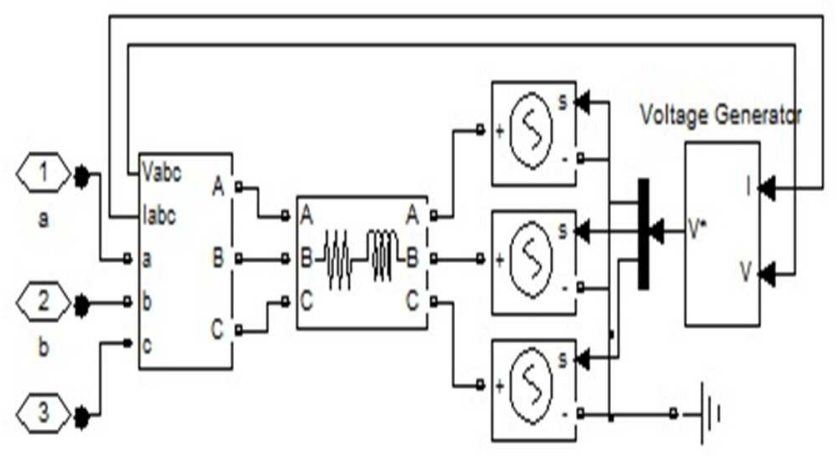

Figure 7. Voltage control generator.

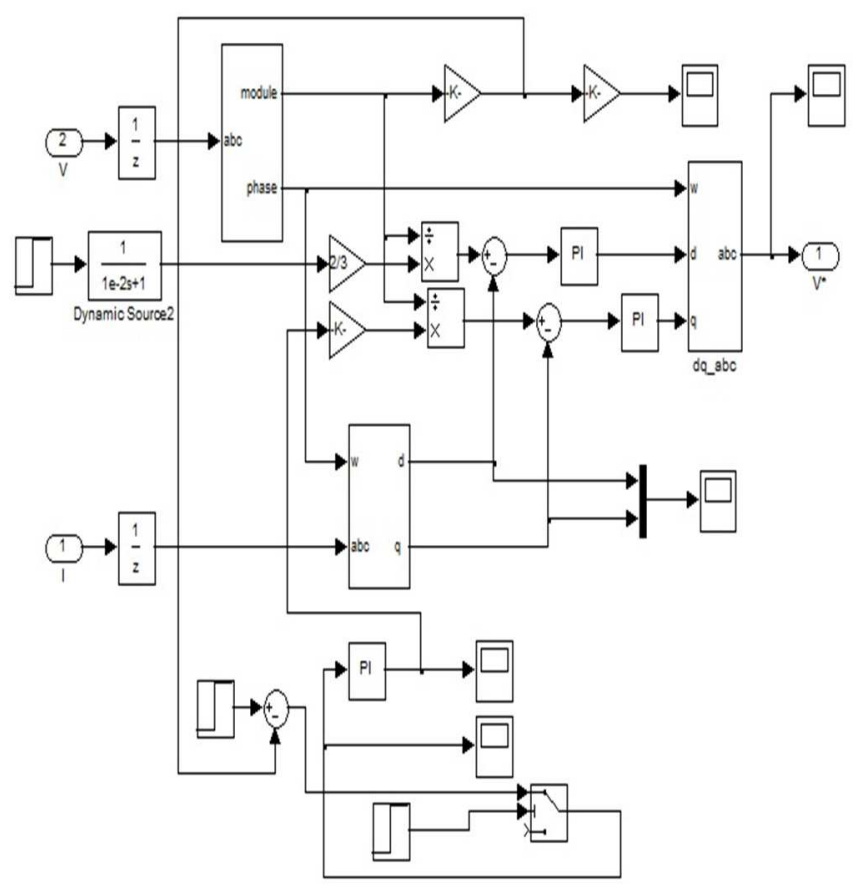

Figure 8. Voltage regulator. 


\subsection{Transmission Line}

This section deals with the modelling of transmission line elements encountered in the electrical power network. Transmission line transmits bulk power from sending to receiving end and represented by standard ( $\pi$ model) consist of four main elements (resistance, inductance, capacitance and conductance). The analysis of power system is mainly dependent on the performance of the transmission line in the power grid. All transmission lines, transformers, and phase shifters are modeled with a common branch model as shown in Figure 9. The three phase series RLC branch block (Figure 10) used to simulate performance of transmission line by setting its parameter corresponding data of network (Milano, 2005).

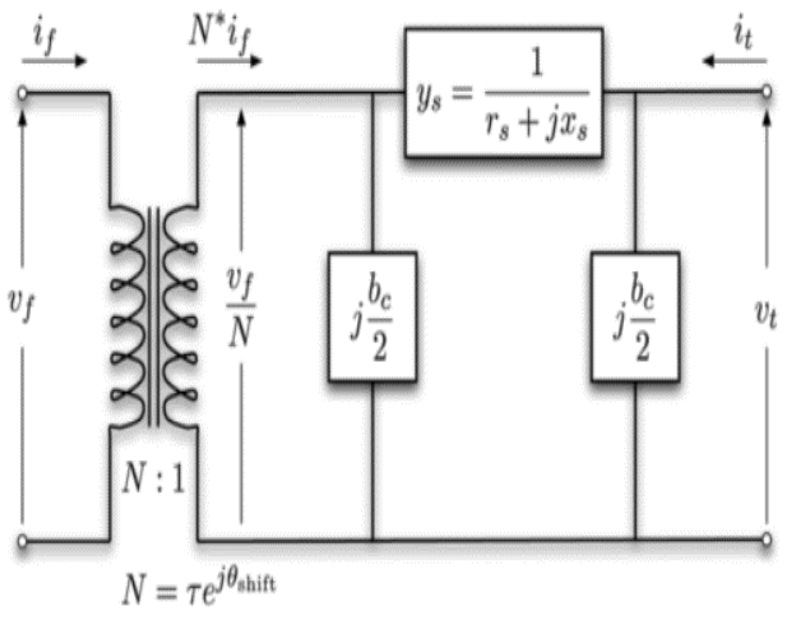

Figure 9. $\pi$ model.

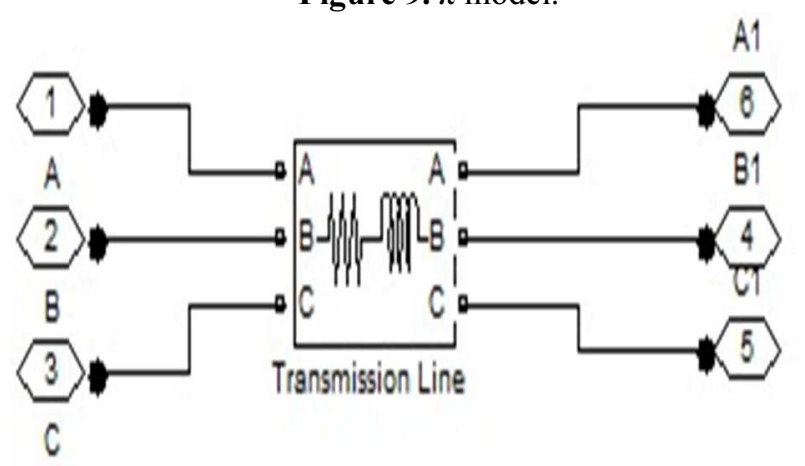

Figure 10. Transmission line simulation.

\subsection{Load bus}

At this bus, the real (Pd) and reactive power load (Qd) are specified and no generator is connected to it. It is required to find out the voltage magnitude and phase angle through load flow calculations. The total demand of the system is represent the distributed loads over the whole network. Power Systems on whether they represent an industrial, commercial or residential load, they can vary greatly in electrical characteristics as well as quantity. In most power simulation, it sees the load as a simplest PQ load characteristic with constant demand of real and reactive power that does not change with any external influences. Various load models have been introduced, taking into account day, month and year cycles as well as it can be consider the voltage and frequency dependencies in the future work. Normally constant power loads are modeled as real and reactive power consumed at a bus (i) as follows:

$$
s_{d}^{i}=p_{d}^{i}+j q_{d}^{i}
$$

The loads can be modeled using Simulink block (three phase series RLC load) but it will be represented as fixed (static) load. A constant MVA load model have no ability to vary with time. Therefore, that, simulation for variable real power load construct with fixed bus voltage reference and variable real power as shown in Figure 19. Fault or any external changes of network state will not effect on load parameters. This load models can be described by the following equation:

$$
\begin{gathered}
P_{d}=P_{o}\left(\frac{V}{V_{o}}\right) \\
Q_{d}=Q_{o}\left(\frac{V}{V_{o}}\right)
\end{gathered}
$$

where $\boldsymbol{Q}_{\boldsymbol{o}}$ stand for reactive powers consumed at reference voltage $\boldsymbol{V}_{\boldsymbol{o}}$ and represented by three phase series RLC load while $\boldsymbol{P}_{\boldsymbol{o}}$ is vary according to load profile curve connected to this model. The external control structure block connected to this model can be variable load curve (continues or discrete). This model will enhance the ability of the system to be studied at different loading conditions.

\section{Simulation Results}

Before modelling the IEEE 5 bus, Newton Raphson method has been implemented to calculate all the parameters of the system. All generation units, load demand and power loss calculated is shown in Table 2.

Table 2. Generation, load demand and losses for IEEE 5bus.

\begin{tabular}{|l|l|}
\hline Unit & Total (MW) \\
\hline Total Generation (PG1,PG2) & 148.05 \\
\hline Total Demand (PD3, PD4, PD5) & 145 \\
\hline Total real power loss & 3.05 \\
\hline
\end{tabular}

The 5 bus IEEE modelling has been developed with Simulink in order to study its behavior under different load conditions. Slack generator simulated to has unlimited real and reactive power generation while its voltage and voltage angel set to $1.06 \angle 0.0^{\circ}$ per unit. Parameters of generation, load, voltage and voltage angel for other buses have set according to data of IEEE five bus shown in Table 3. 
Table 3. IEEE 5 Bus Data.

\begin{tabular}{|l|l|l|l|l|l|l|l|}
\hline \multirow{2}{*}{$\begin{array}{l}\text { Bus } \\
\text { No. }\end{array}$} & \multirow{2}{*}{ Type } & \multicolumn{2}{l|}{ Generation } & \multicolumn{2}{l|}{ Load } & \multicolumn{2}{l|}{ Voltage } \\
\cline { 3 - 8 } & & Real & React. & Real & React. & Magn. & angle \\
\hline 1 & 1 & 0 & 0 & 0 & 0 & 1.06 & 0 \\
\hline 2 & 2 & 90 & 0 & 0 & 0 & 1 & 0 \\
\hline 3 & 0 & 0 & 0 & 45 & 15 & 1 & 0 \\
\hline 4 & 0 & 0 & 0 & 40 & 5 & 1 & 0 \\
\hline 5 & 0 & 0 & 0 & 60 & 10 & 1 & 0 \\
\hline
\end{tabular}

At the beginning, load is considered as constant and simulation implemented for 10 seconds to compare with Newton Raphson (NR) method results. Figure 11 and Figure 12 shows measurements of real power flow (RPF) and losses (RPL), respectively, in all the transmission lines. This measurements has been compared with accurate calculated by Newton Raphson method to valid Simulink model as in Table 4.

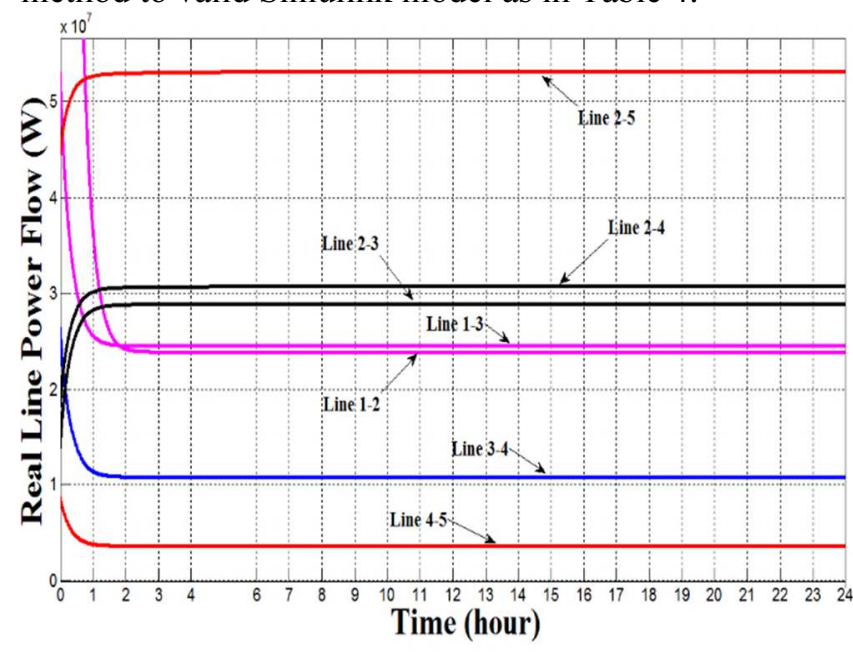

Figure 11. Active power flow on the lines with constant load.

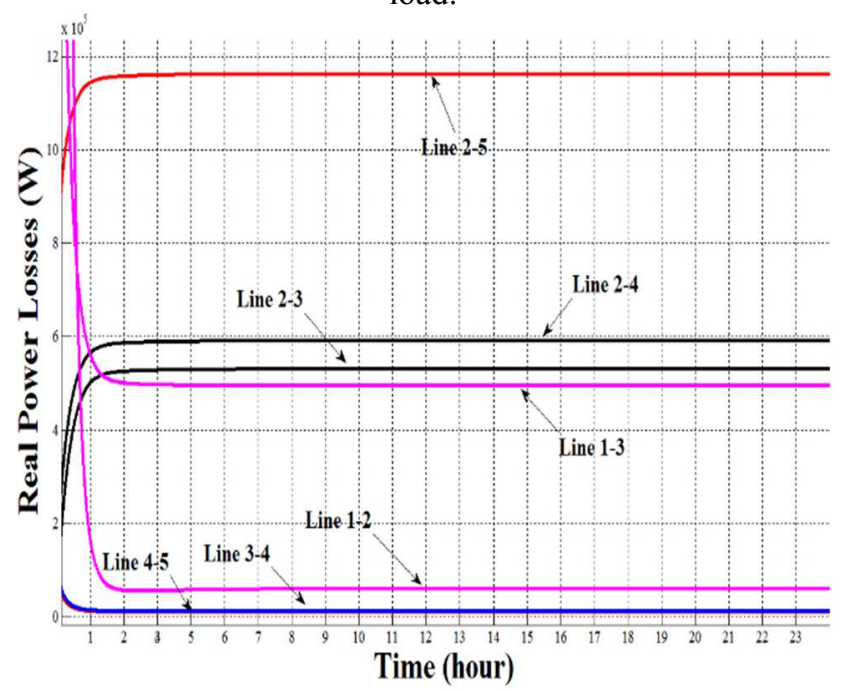

Figure 12. Active power losses on the lines with constant load.
In order to validate the proposed variable load simulation, random load profile used as input signal to Figure 19 instead of constant one. In Figure 13, output and output signals are identical except very small transient when signal switch from value to another.

Table 4. Real power lines flow and losses by NR.

\begin{tabular}{|l|c|c|c|c|c|c|c|}
\cline { 2 - 8 } \multicolumn{1}{c|}{} & \multicolumn{7}{|c|}{ Transmission Lines } \\
\cline { 2 - 8 } & $1-2$ & $1-3$ & $2-3$ & $2-4$ & $2-5$ & $3-4$ & $5-4$ \\
\hline$R P F(M W)$ & 15.09 & 27.05 & 30.50 & 32.60 & 56.06 & 11.76 & 3.97 \\
\hline$R P L(M W)$ & 0.069 & 0.55 & 0.525 & 0.594 & 0.429 & 0.013 & 0.013 \\
\hline
\end{tabular}
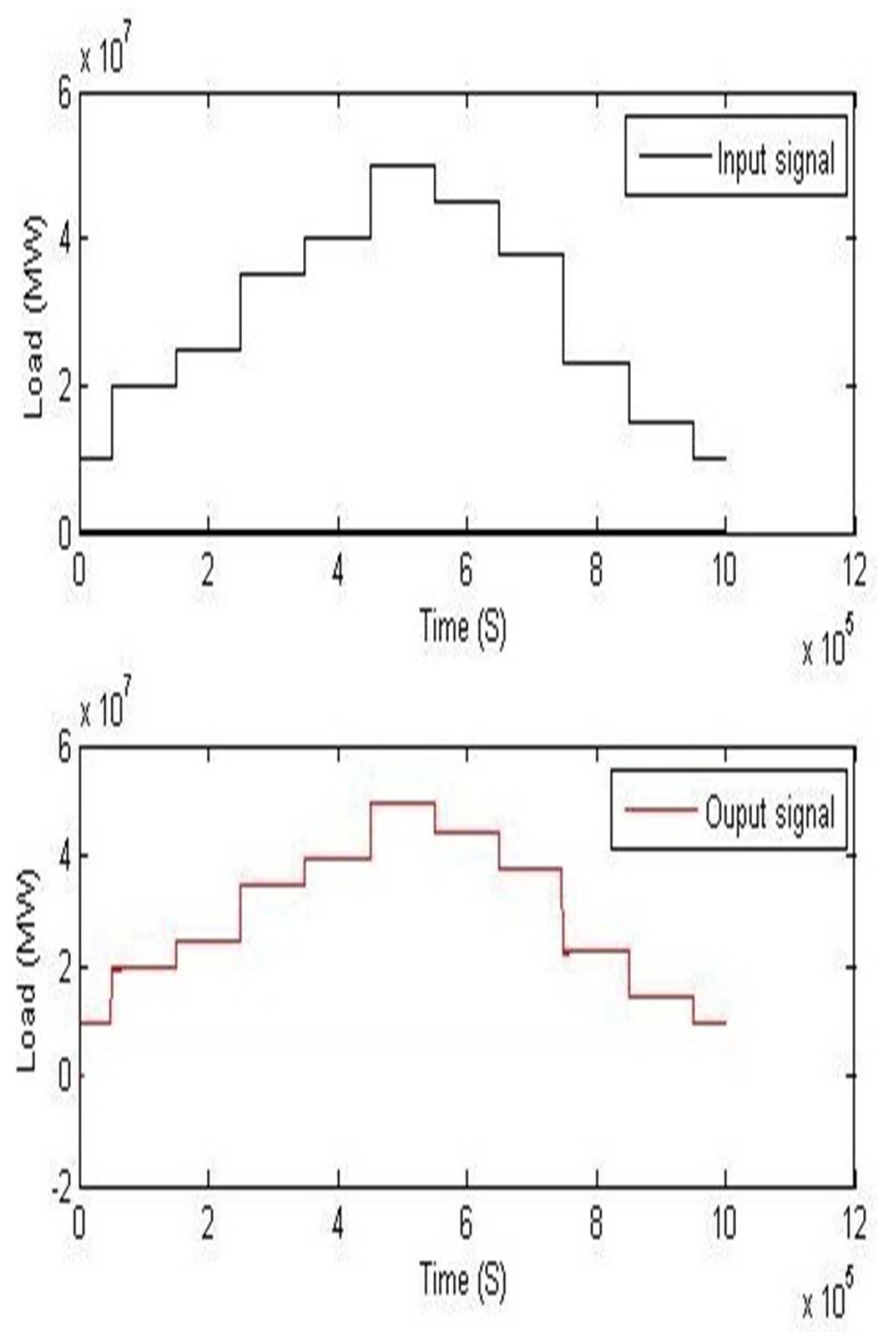

Figure 13. Load model testing.

Three different daily load profile (residential, commercial and industrial) are connected to load buses as follows: residential load curve (Figure 14) connect to bus 3, commercial load curve (Figure 15) connect to bus 4 and industrial load curve (Figure 16) connect to bus 5 . 


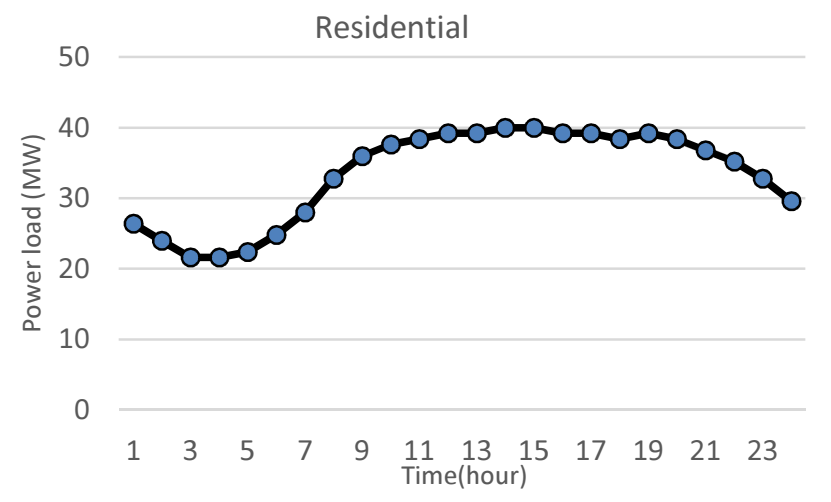

Figure 14. Residential daily load curve connect to bus 3 .

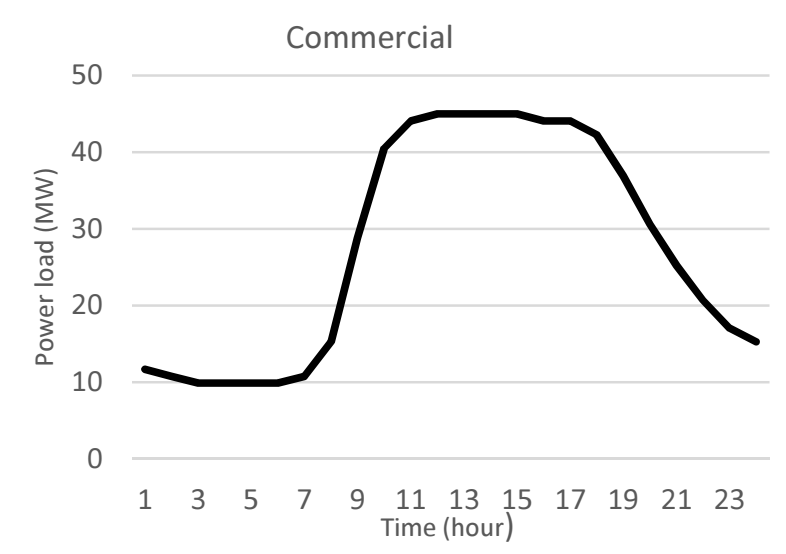

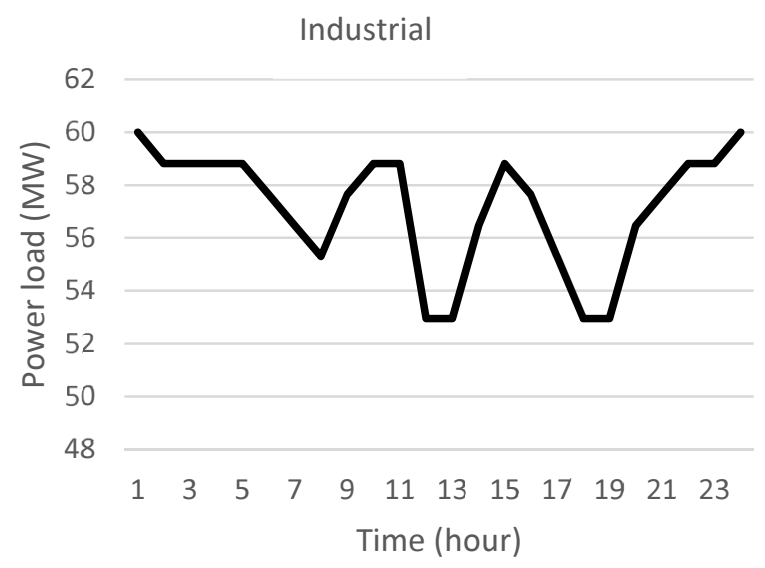

Figure 16. Industrial daily load curve connect to bus 5 .

The results show that real power flow and losses in transmission lines will be effect by variable load profiles as shown in Figure 17 and Figure 18. The simulation of variable load details in Figure 19. The 5 bus Simulink simulation under different load conditions is performed on a computer with core i74800MQ CPU, $2.7 \mathrm{GHz}$, 16GB RAM and Microsoft Windows 7 operating system running in real-time mode.

Figure 15. Commercial daily load curve connect to bus 4 .

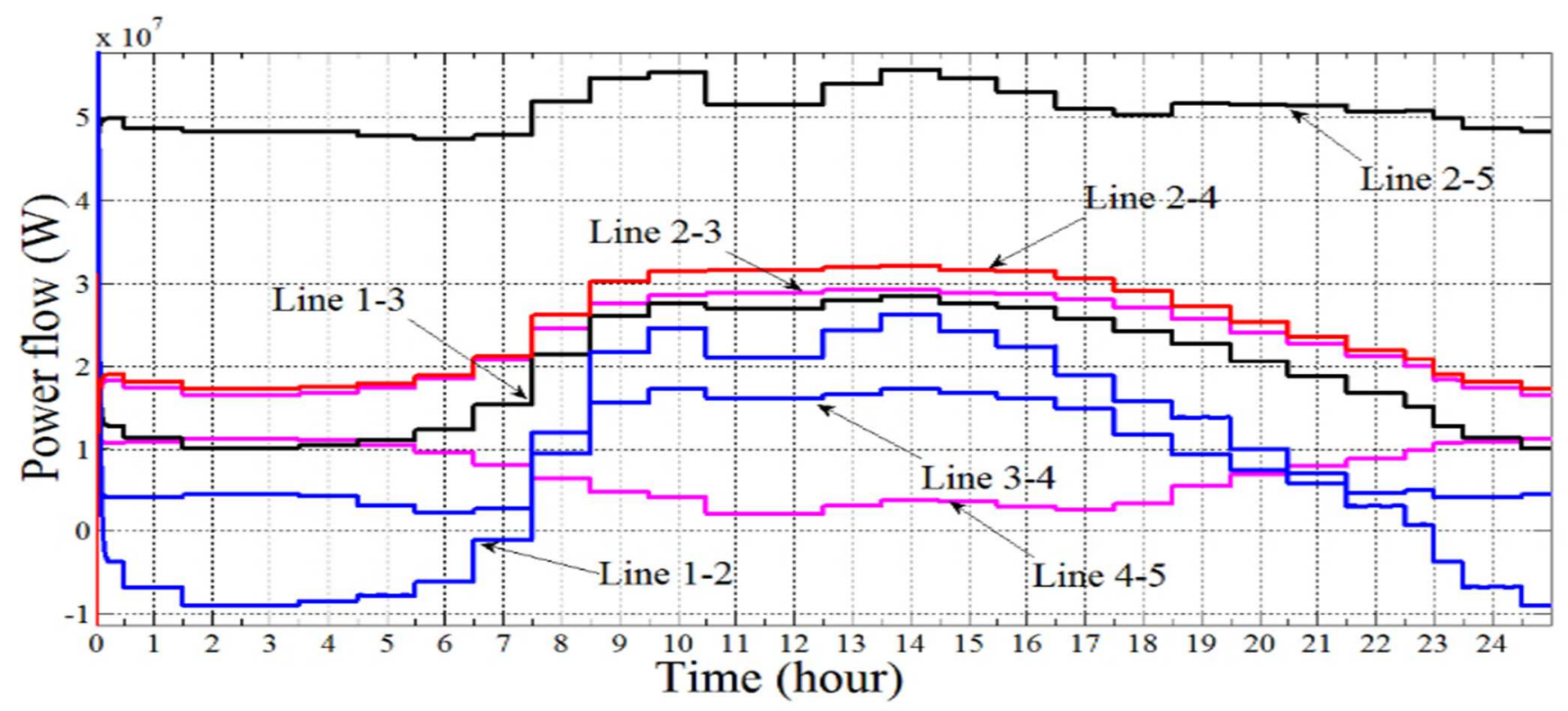

Figure 17. Lines Power flow with variable loads. 


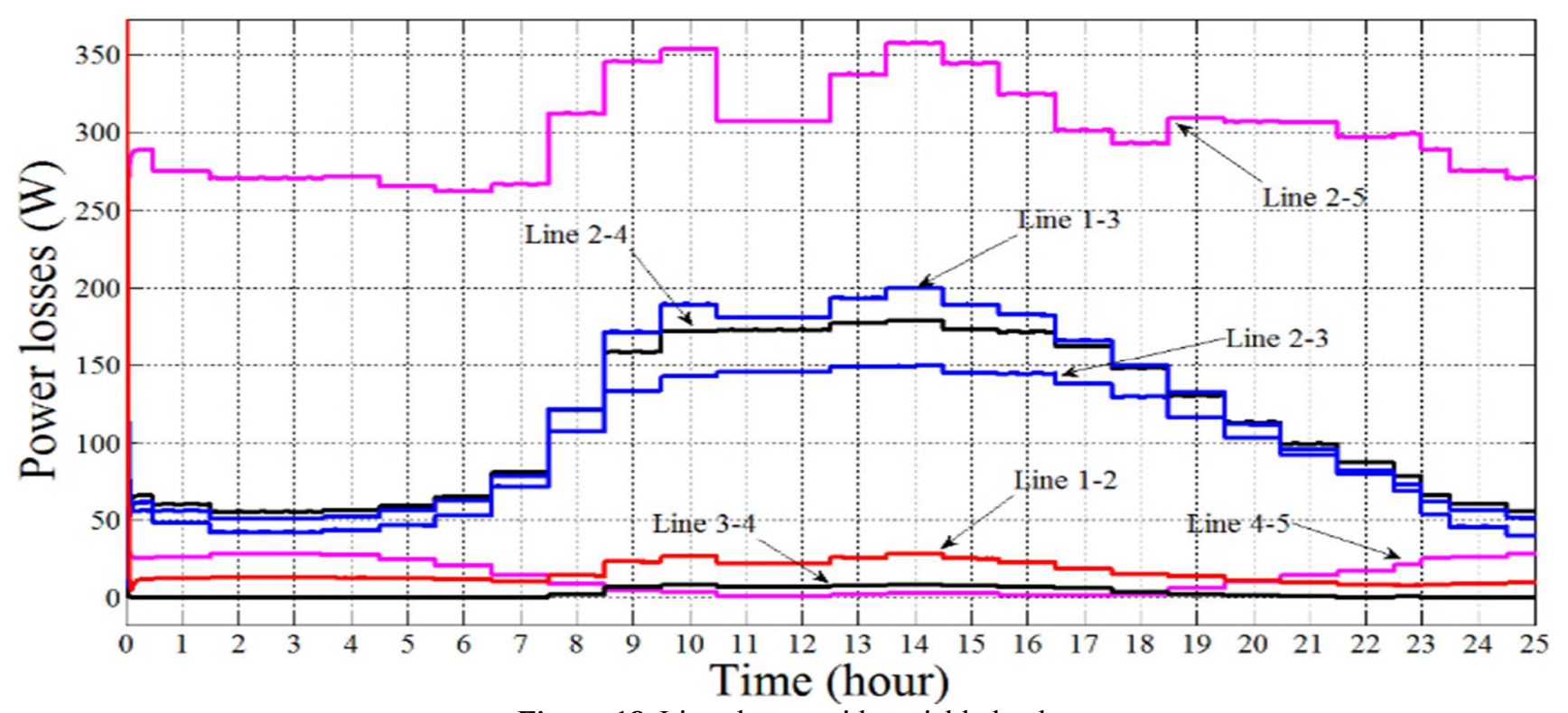

Figure 18. Lines losses with variable loads.

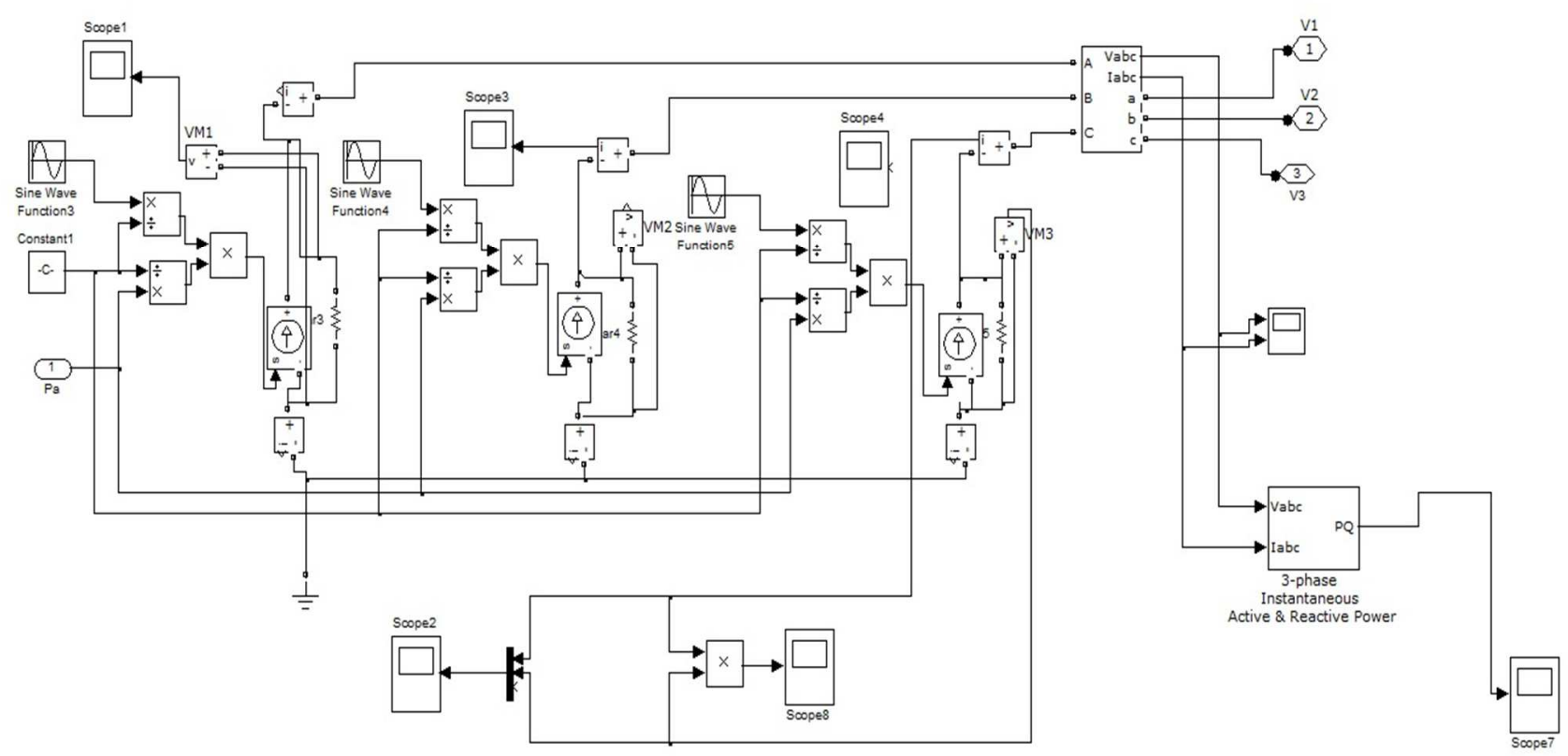

Figure 19. Variable load simulation.

\section{Conclusions}

This paper has presented network modelling with variable load profile using Simulink. . System of IEEE five bus is used as a test bed. This simulation can be easily adapted to accommodate different types of generation resources by considering time-varying load profile. The simulation introduce four main blocks represent slack generator, control generator, transmission line and variable load. Three different types of load profile (residential, commercial and industrial) tested the five bus network simulation. The results demonstrate that the simulation can be adequate to identify the real power flow and losses into transmission lines. Simulation results indicate that varying demand can change the dynamic performance of the system and will help DNOs understand what they need to do to provide solutions for network stability.

\section{References}

Katia C. Almeida and Roberto Salgado. Optimal Power Flow Solutions Under Variable Load Conditions. IEEE Transactions on Power systems, 15(4): 1204 - 1211, 2000.

Mohammadhafez Bazrafshan and Nikolaos Gatsis. Decentralized Stochastic Optimal Power Flow in Radial Networks With Distributed Generation. IEEE Transaction on Smart Grid, 8(2): 787 - 801, 2017. 
R. F. Chang and C. N. Lu. Load profiling and its applications in power market. In IEEE Power Engineering Society General Meeting, Toronto, 2003.

G. Geng, J Liang, R. G. Harley, and R. Qu. Load Profile Partitioning and Dynamic Reactive Power Optimization International. In Conference on Power System Technology (POWERCON), pp. 1-8, Hangzhou, 2010.

Swaroop S. Guggilam, Emiliano Dall'Anese, Yu Christine Chen, Sairaj V. Dhople, and Georgios B. Giannakis. Scalable Optimization Methods for Distribution Networks with High PV Integration. IEEE Transactions on Smart Grid, 7(4): 2061-2070, 2016.

Ignacio Hernando-Gil, Irinel-Sorin Ilie, and Sasa Z. Djokic. Reliability planning of active distribution systems incorporating regulator requirements and networkreliability equivalents. IET Generation, Transmission \& Distribution, 10(1): 93 - 106, 2016.

J.A. Jardini, C.M.V. Tahan, M.R. Gouvea, S.U. Ahn, and F.M. Figueiredo. Daily Load Profiles for Residential, Commercial and Industrial Low Voltage Consumers. IEEE Transactions on Power Delivery, 15(1):375 - 380, 2000.

Sun Ic Kim, Hae Soon Kim, Yong Jae Joo, and Ji Hyun Kim. Power usage pattern and consumption separation method by load devices based on remote metering system's Load profile data. In 11th International Conference on Control, Automation and Systems (ICCAS), Gyeonggi-do, 2011.

Willis H. Lee. Power Distribution Planning Reference Book, Second Edition, Revised and Expanded. New York : Marcel Dekker, Inc., 2004.

Ran Li, Furong Li, and Nathan D. Smith. Multi-Resolution Load Profile Clustering for Smart Metering Data. IEEE Transactions on Power Systems, 31(6): 4473-4482, 2016.

Jinghuan Ma, He Henry Chen, Lingyang Song, and Yonghui Li. Residential Load Scheduling in Smart Grid: A Cost Efficiency Perspective. IEEE Transaction on Smart Grid, 7(2): 771 - 784, 2016.

F. Milano. An open source power system analysis toolbox. IEEE Transactions on Power systems, 20(3): 1199-1206, 2016.

Christos Thrampoulidis, Subhonmesh Bose, and Babak Hassibi. Optimal Placement of Distributed Energy Storage in Power Networks. IEEE Transactions on Automatic Control, 61(2): 416 - 429, 2016.

Ervin D. Varga, Christian Noce, and Gianluca Sapienza Robust Real-Time Load Profile Encoding and Classification Framework for Efficient Power Systems Operation. IEEE Transaction on Power Systems, 30(4): 1897 - 1904, 2015.

Jialiang Yi, Pádraig F. Lyons, Peter J. Davison, Pengfei Wang, and Philip C. Taylor. Robust Scheduling Scheme for Energy Storage to Facilitate High Penetration of Renewables. IEEE Transaction on Sustainable Energy, 7(2):797 - 807, 2016. 